\title{
Extrahepatic Portal Venous Obstruction With Hepatic Enzyme Elevation Resembling Hepatitis in Patients With Cancer
}

\author{
TZU-YAO LIAO ${ }^{1}$, CHUANG-CHI LIAW ${ }^{1}$, HUI-CHING HSU ${ }^{2}$, CHIA-HSUN HSIEH ${ }^{1}$, \\ JOHN WEN-CHENG CHANG ${ }^{1}$ and YU-HSIANG JUAN ${ }^{3}$ \\ ${ }^{1}$ Division of Hemato-Oncology, Department of Internal Medicine, \\ Chang-Gung Memorial Hospital and Chang-Gung University College of Medicine, Taoyuan, Taiwan, R.O.C.; \\ ${ }^{2}$ Division of Chinese Acupuncture and Traumatology, Department of Traditional Chinese Medicine, \\ Chang-Gung Memorial Hospital and Chang-Gung University College of Medicine, Taoyuan, Taiwan, R.O.C.; \\ ${ }^{3}$ Department of Medical Imaging and Intervention, \\ Chang-Gung Memorial Hospital and Chang-Gung University College of Medicine, Taoyuan, Taiwan, R.O.C.
}

\begin{abstract}
Background/Aim: Chemotherapy is often halted due to abnormal liver function resembling hepatitis. But the cause can be extrahepatic portal venous obstruction (EHPVO) with hepatic enzyme elevation rather than being an adverse effect of chemotherapy. We investigated EHPVO with hepatic enzyme elevation in patients with cancer. Patients and Methods: Data of these hospitalized patients with solid tumors between January 2013 and September 2017 were collected. The criteria for study inclusion were: (i) Extrahepatic malignancy; (ii) computed tomographic scans showing a tumor with external compression of the extrahepatic portal vein; and (iii) serum aminotransferase (AST) or alanine transaminase (ALT) level three times above the normal value. Results: Thirteen out of 377 (3\%) patients developed EHPVO with hepatic enzyme elevation, as demonstrated from computed tomographic scan. Four cases (31\%) also had vascular thrombosis (three portal vein and one inferior vena cava). Serum AST increased from $34 \pm 11$ to $169 \pm 94$ U/l. ALT increased from $9 \pm 38$ to $177 \pm 104$ U/l. There was no relationship of EHPVO with viral markers and cirrhosis. Six cases received chemotherapy with liver function improvement. Conclusion: EHPVO occurred in
\end{abstract}

This article is freely accessible online.

Correspondence to: Chuang-Chi Liaw, MD, Division of HematoOncology, Department of Internal Medicine, Chang-Gung Memorial Hospital, 5, Fusing St., Gueishan Township, Taoyuan City, 333 , Taiwan, R.O.C. Tel: +886 33281200 Ext. 8825, Fax: +886 3278211, e-mail: e102309@adm.cgmh.org.tw

Key Words: Extrahepatic portal vein obstruction, hepatic enzyme elevation, computed tomography, thromboembolic complications. patients with metastatic cancer, leading to hepatic enzyme elevation resembling hepatitis without hepatitis risk factors and cirrhosis. Before withholding chemotherapy due to hepatic enzyme elevation, the possibility of EHPVO should firstly be excluded.

Chemotherapy is often halted due to abnormal liver function resembling hepatitis. But the cause can be extrahepatic portal venous obstruction (EHPVO) with hepatic enzyme elevation rather than being an adverse effect of chemotherapy. The portal vein is located in the right upper quadrant of the abdomen, originating behind the upper edge of the pancreatic head (1). The portal venous system is responsible for draining the blood from the spleen and parts of the gastrointestinal tract to the liver (1). The superior and inferior mesenteric veins join the splenic vein form the portal vein (1). EHPVO is a hepatic vascular disorder which results in obstruction and cavernomatous transformation of the portal vein outside the liver, which may or may not involve intrahepatic portal vein, splenic vein, or mesenteric vein. Cirrhosis of the liver and neoplasms in adults are the most common etiology of EHPVO (2-4). Such tumors can cause compression or direct invasion of the portal vein and lead to thrombosis by inducing a hypercoagulable state. The development of collateral circulation also has the risk of varices bleeding (2-4). Hepatic enzyme elevation represents liver injury; such hepatic dysfunction is not uncommon in patients with EHPVO. Liver transaminases (5), including aspartate aminotransferase (AST) and alanine transaminase (ALT), are two of the most useful measures of liver injury (6).

In this case series study, we investigated EHPVO with hepatic enzyme elevation in patients with cancer. Findings of their abdominal computed tomography (CT) scans and management were also discussed. 
in vivo $33: 1697-1702(2019)$

Table I. Characteristics of the 13 patients with cancer with extrahepatic portal vein obstructions with hepatic enzyme elevation.

Characteristic

Age, years

Gender, n

Primary site, n (\%)

Performance status, n (\%)

Associated with other thromboembolic complications, n (\%)

Associated with other paraneoplastic syndrome, n (\%)
Median (range)

Male/female

No./total hospitalized

Urothelial carcinoma

Non-urothelial carcinoma

$0-1$

$\geq 2$

Yes

No

Yes

No
$64(32-82)$ $9 / 4$

13/377 (3)

$7 / 13(54)$

6/13 (46)

$7 / 13(54)$

$6 / 13(46)$

$10 / 13(77)$

$3 / 13(23)$

9/13 (69)

$4 / 13(31)$

\section{Patients and Methods}

This study was approved by the Institutional Review Board of Chang Gung Medical Foundation on 2017/11/02. The IRB is organized and operates according to the guidelines of good clinical practice and the applicable laws and regulations (no. 201701668B0).

Study population. Between January 2013 and September 2017, we conducted a retrospectively case series study using data collected from 377 patients admitted to the Oncology wards of Chang-Gung Memorial Hospital, Taoyuan, Taiwan, ROC. A single physician provided most of the data. The physician's field of expertise was urological malignancies. Thirteen patients (3\%) with EHPVO with hepatic enzyme elevation were identified. Diagnosis was based on CT findings and worsening of liver enzyme AST and ALT levels. The criteria for inclusion in the study were: (i) Extrahepatic malignancy; (ii) CT scans showing a tumor with external compression of the extrahepatic portal vein; and (iii) Presence of AST or ALT three times above normal value (AST $\leq 34 \mathrm{U} / \mathrm{I}$ and ALT $\leq 36 \mathrm{U} / \mathrm{I})$.

For the purpose of the study of EHPVO, CT scan rather than magnetic resonance imaging was used in our clinical practice. CT scans from axial and coronal view demonstrated tumor location, portal vein external compression site with or without association thrombosis. Hepatic function including serum AST, ALT and bilirubin were monitored. Serum gamma-glutamyl transpeptidase and ammonia were checked in selected patients. Hepatic enzyme elevation was defined as three times the upper limit of normal. Hepatic enzyme improvement was defined as both AST and ALT returning to normal or near normal values. Other laboratory data and clinical important findings were: (i) D-dimer test results; (ii) complete blood counts; (iii) activated partial thromboplastin time (APTT), prothrombin time (PT) and C-reactive protein (CRP) in selected patients; (iii) other thromboembolic complications; and (iv) other paraneoplastic syndromes. We applied a cut-off value for the D-dimer test results of $500 \mathrm{ng} / \mathrm{ml}$. Common thromboembolism-associated complications included symptoms of iliofemoral vein obstruction, pulmonary venous obstruction and cerebral thrombosis. Common paraneoplastic syndromes included cachexia syndrome (simultaneous weight loss of more than 5\% within 6 months, reduced food intake, and muscle wasting), hypercalcemia (serum calcium level $>11 \mathrm{mg} / \mathrm{dl}$ ), leukemoid reaction (peripheral white cell count $>20,000 / \mu l$ without evidence of infection or leukemia), and prerenal azotemia (defined as a blood urea nitrogen $(\mathrm{BUN}) /$ creatinine ratio $>20)$.

Treatment was systemic therapy. For thromboembolic complications, low-molecular-weight heparin (LMWH, Clexane injection, Enoxaparin; Sanofi-Aventis, France) as a subcutaneous injection. Intravenous dexamethasone, and intravenous fluids were also given in most cases (8). CT scans were obtained from the Hospital Picture Archiving and Communication System.

Statistical methods. Continuous serum D-dimer, AST and ALT level data (presented as mean \pm standard deviation) were gathered, and survival time was calculated from the time of the diagnosis of an iliofemoral venous obstruction to the patient's death. Survival curves were determined using the Kaplan-Meier method. Chisquared test was used adding Fisher's exact test due to small sample size to detect differences between subgroups, and a $p$-value less than 0.05 was considered statistically significant.

\section{Results}

The patients' clinical characteristics are shown in Table I. We had 13 consecutive patients with cancer who developed EHPVO with hepatic enzyme elevation, including nine men and four women (55-82 years old; median age: 64 years). These patients had various types of metastatic tumors, one case of which was detected at initial breast cancer diagnosis. Urothelial carcinoma was the most common diagnosis, of the urinary bladder in four, ureter in two and renal pelvis in one. Other malignancies included renal cell carcinoma in one, lung in one, stomach in one, pancreas in one esophagus in one and breast in one. Six patients had an Eastern Cooperative Oncology Group (ECOG) performance status of two or greater. Ten patients had other thromboembolic complications, nine had paraneoplastic syndromes. Common thromboembolic complications included iliofemoral vein obstruction/thrombophlebitis (in four), cerebral thrombosis (in three) and pulmonary venous obstruction (in three). Common paraneoplastic syndromes were cachexia syndrome (in six) with combination with hypercalcemia (in one), and leukemoid reaction (in three). 
Important laboratory and imaging findings of 13 patients with cancer with EHPVO with hepatic enzyme elevation are listed in Table II. All patients had different degrees of malaise and fatigue. D-Dimer levels and complete blood counts were measured for all patients. The mean D-dimer value was $3,647 \pm 1,884 \mathrm{ng} / \mathrm{ml}$ (863 to $>10,000 \mathrm{ng} / \mathrm{ml}$ ). DDimer values over $3000 \mathrm{ng} / \mathrm{ml}$ were recorded for nine of the patients. There were five patients with hemoglobin levels below $10 \mathrm{~g} / \mathrm{dl}$, six with elevated white blood cell count $(>10,000 / \mu \mathrm{l})$, and two with decreased platelet count $(<100,000 / \mu \mathrm{l})$. APTT and PT were assessed in seven patients. Of these, two had APTT values $>36 \mathrm{~s}$, and 3 had PT values $>15 \mathrm{~s}(43 \%)$. Albumin values were $<3.0 \mathrm{~g} / \mathrm{dl}$ in three out of the nine patients. CRP was monitored in 5 patients. The mean CRP value was $75 \pm 60 \mathrm{mg} / \mathrm{l}$ (17.7-157.1 mg/l). Prerenal azotemia was assessed in 13 patients. Of these, five (38\%) had BUN/creatinine ratio $>20$.

Serum AST and ALT monitored during, before and after EHVPO with liver enzyme elevation. During hepatic enzyme elevation, serum AST was 169 \pm 94 U/1 (47-325 U/l). and serum ALT were $177 \pm 104 \mathrm{U} / 1$ (47-419 U/1). Bilirubin was checked in 12 patents, four had elevation $(>1.2 \mathrm{mg} / \mathrm{dl})$. Gamma-glutamyl transferase was checked in four patients and found to be elevated (normal value $<36 \mathrm{IU} / 1$ ), with serum value of $816 \pm 580 \mathrm{IU} / 1$ (12-388 IU/1). Serum ammonium was checked in two patents, with elevation (normal value $<80 \mathrm{ug} / \mathrm{dl}$ ). Hepatitis $\mathrm{B}$ antigen was monitored in 11 patients, and was positive in to cases. Antibody to hepatitis $\mathrm{C}$ was monitored in 11 patients, all were negative. No patients were found to have liver cirrhosis. Three cases had liver metastases. Based on statistical methods with Fisher's exact test, they were no relationship with viral markers, cirrhosis and liver lesions.

Before hepatic enzyme elevation, serum AST and ALT were checked in 12 patients. The value of AST was $34 \pm 11 \mathrm{U} / 1$ (17-52 U/1) and serum ALT was 9 \pm 38 U/1 (8-146 U/l). Followup hepatic enzyme in their course, serum AST was check in 12 patients, six showed serum hepatic enzyme improvement (less than 1.5 times above normal value).

All patients from CT scans exhibited EHPVO by lesions. CT scans from axial view can detect lesions behind pancreas anterior to the inferior vena cava (IVC) and aorta. CT scans from coronal views can detect lesions with obstruction level: A bladder tumor with EHPVO by tumor and demonstrating portal vein thrombosis (Figure 1); A bladder tumor with EHPVO by tumor, without detecting vascular thrombosis (Figure 2); A breast cancer elevation of hepatic enzymes initially with EHPVO by tumor, without detecting vascular thrombosis (Figure 3); Esophageal squamous cell carcinoma elevated serum hepatic enzyme before treatment, his CT scan from coronal and axial view showed EHPVO by tumor with collateral circulation formation, demonstrating portal vein and inferior vena cava thrombosis (Figure 4).
Table II. Important laboratory and imaging findings of 13 patients with cancer with extrahepatic portal vein obstruction with hepatic enzyme elevation

\begin{tabular}{|c|c|c|}
\hline Characteristic & $\mathrm{n}(\%)$ & $p$-Value \\
\hline \multicolumn{3}{|c|}{$\begin{array}{l}\text { Detection of HBsAg or } \\
\text { anti-HCV, } n(\%)\end{array}$} \\
\hline Yes & $2 / 12(17)$ & 0.003 \\
\hline No & $10 / 12(83)$ & \\
\hline \multicolumn{3}{|l|}{ Liver cirrhosis, n (\%) } \\
\hline Yes & $0 / 13(0)$ & $0.001^{\#}$ \\
\hline No & $13 / 13(100)$ & \\
\hline \multicolumn{3}{|c|}{ Presence of liver lesion, n (\%) } \\
\hline Yes & $3 / 13(23)$ & 0.02 \\
\hline No & $10 / 13(77)$ & \\
\hline \multicolumn{3}{|c|}{ Abnormal hepatic enzyme, n (\%) } \\
\hline Before therapy & $5 / 13(39)$ & $0.24^{*}$ \\
\hline After therapy & $8 / 13(61)$ & \\
\hline \multicolumn{3}{|c|}{$\begin{array}{l}\text { Associated with vascular } \\
\text { thrombosis, } \mathrm{n}(\%)\end{array}$} \\
\hline Yes & $4 / 13(31)$ & $0.05^{*}$ \\
\hline No & $9 / 13(69)$ & \\
\hline \multicolumn{3}{|l|}{ D-Dimer, ng/ml } \\
\hline Median (IQR) & $3,664(365-6,963)$ & $0.05^{*}$ \\
\hline$\leq 3,000$ & 4/13 (31) & \\
\hline$>3,000$ & $9 / 13(69)$ & \\
\hline \multicolumn{3}{|l|}{ AST at diagnosis, $\mathrm{U} / 1$} \\
\hline Median (IQR) & $153(103-203)$ & $<0.0001$ \\
\hline Elevation & $13 / 13(100)$ & \\
\hline Without elevation & $0 / 13(0)$ & \\
\hline \multicolumn{3}{|l|}{ ALT at diagnosis, $\mathrm{U} / \mathrm{l}$} \\
\hline Median (IQR) & $150(136-164)$ & $<0.0001$ \\
\hline Elevation & $13 / 13(100)$ & \\
\hline Without elevation & $0 / 13(0)$ & \\
\hline
\end{tabular}

AST: Aminotransferase; ALT: alanine transaminase; HBsAg: hepatitis B antigen; anti-HCV: antibody hepatitis C; IVC: inferior vena cava. IQR: interquartile range. "Fisher's exact test; ${ }^{*}$ Chi-squared test.

Treatments included underlying disease treatment, enoxaparin, intravenous dexamethasone, and intravenous fluids for the complication of coagulopathy. Ten patients received systemic therapy. including chemotherapy in nine, and targeted therapy in six cases received chemotherapy with liver function improvement. Nine patients were given LMWH with/without dexamethasone due to thromboembolic complications. Their follow-up period ranged from 2 to 27 weeks. All patients were followed-up until death or the present. Only one patient with breast cancer was alive at the time of writing. Median overall survival time by Kaplan-Meier methods was 8 weeks. The predeath status in these patients was loss of consciousness.

\section{Discussion}

EHPVO with liver enzyme elevation compromised $3 \%$ of our hospital's cases of malignancies. All patients had metastatic malignant disease. EHPVO can be found at initial 
A

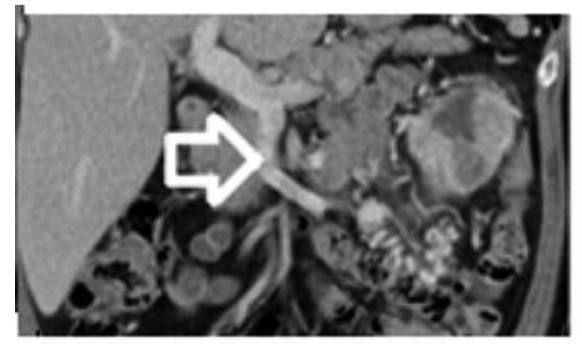

B

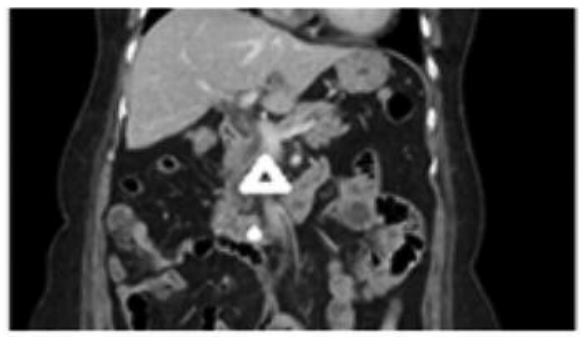

C

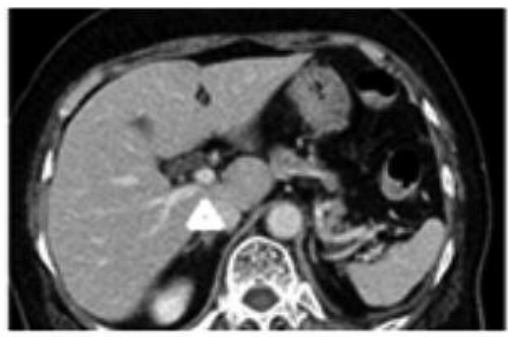

Figure 1. Extrahepatic portal vein obstruction (EHPVO) with hepatic enzyme elevation. An 82-year-old woman with bladder urothelial carcinoma underwent chemotherapy. She had hepatic enzyme elevation with aminotransferase levels of $340 \mathrm{U} / \mathrm{l}$ and alanine transaminase levels of 419 U/l. A and B: Coronal view in computed tomographic scan showed EHPVO by tumor with portal vein thrombosis. C: Axial view showed a mass anterior to the inferior vena cava. Arrowheads indicate the lesions with EHPVO. Arrow indicates thrombotic lesions.

A

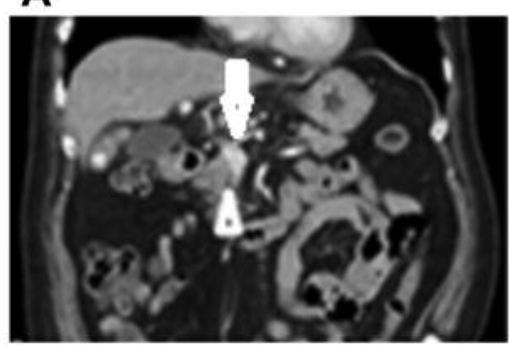

B

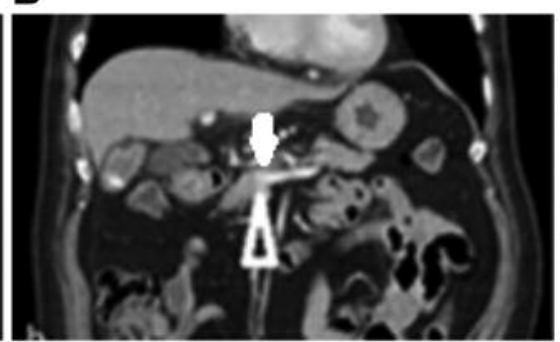

C

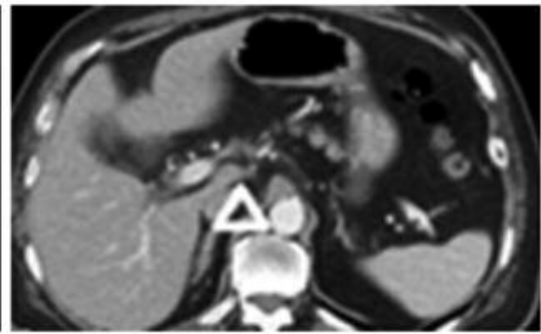

Figure 2. Extrahepatic portal vein obstruction (EHPVO) with hepatic enzyme elevation. A 64-year-old man with bladder urothelial carcinoma underwent chemotherapy. He had hepatic enzyme elevation with aminotransferase levels of 283 U/l and alanine transaminase levels of 252 U/l. A and B: Computed tomographic scan in coronal view showed EHPVO by tumor. C: Axial view showed a mass anterior to the inferior vena cava and aorta. Arrowheads indicate the lesions with EHPVO. Arrows indicate extrahepatic portal vein.

A

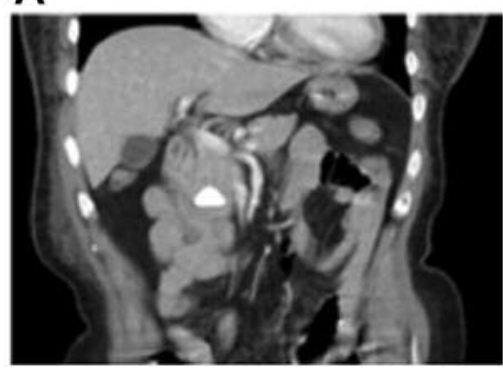

B

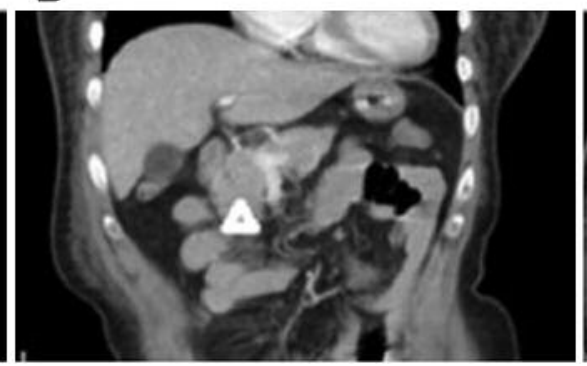

C

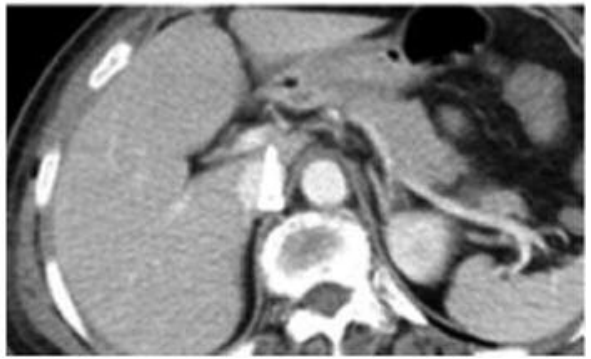

Figure 3. Extrahepatic portal vein obstruction (EHPVO) with hepatic enzyme elevation. A 59-year-old woman with breast cancer underwent chemotherapy. Before chemotherapy, she initially presented hepatic enzyme elevation with aminotransferase levels of 127 U/l and alanine transaminase levels of 79 U/l. A: Coronal view on computed tomographic scan showed EHPVO by tumor. B: Axial view of the mass anterior to the inferior vena cava and aorta. C: Axial computed tomographic (CT) revealed a mass anterior to the inferior vena cava and aorta. Arrowheads indicate the lesions with EHPVO.

cancer diagnosis They are also frequently associated with other thromboembolic complications and patients exhibited other paraneoplastic syndromes. EHPVO demonstrated no relationship with viral markers, cirrhosis and liver lesions. Like iliofemoral vein obstruction (7), EHPVO is a vascular disorder, it is also a thromboembolic complication.
The tumors that cause EHPVOs usually involve lesions behind the pancreas. The route of spreading is similar to that of the portal venous drainage system. In addition to pancreatic cancer, low esophageal and gastric cancer caused the EHPVOs in our study. Urothelial carcinomas were a common cause of HPVO in our patients. Urothelial 
A

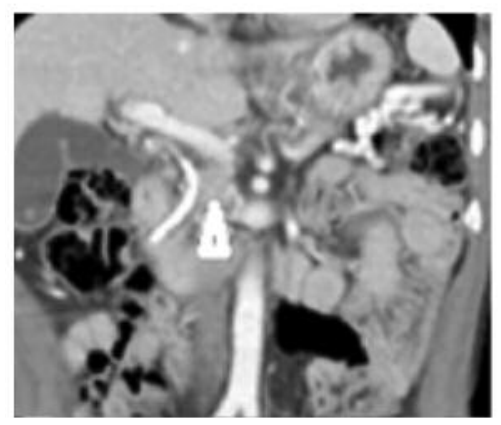

B

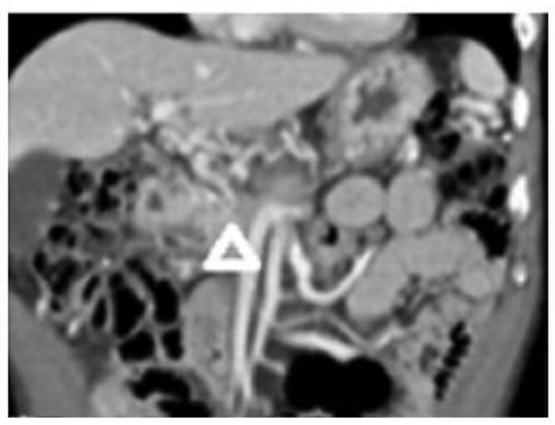

C

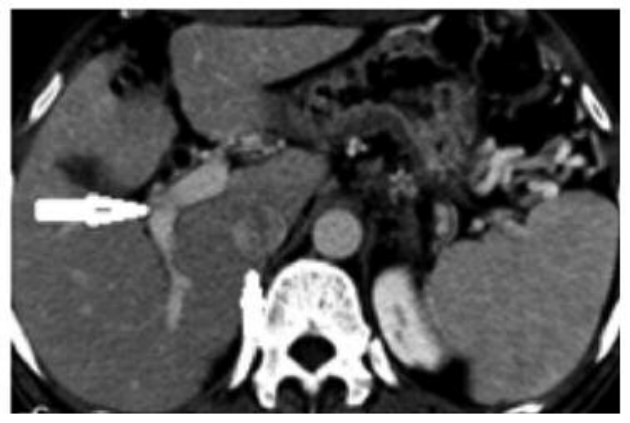

Figure 4. Extrahepatic portal vein obstruction (EHPVO) with hepatic enzyme elevation. A 56-year-old man with squamous cell carcinoma underwent chemotherapy. Before chemotherapy, hepatic enzyme levels were elevated with aminotransferase levels of 47 U/l and ALT 177 U/l. A and B: Computed tomographic scan in coronal view (B) showed EHPVO by tumor with formation of collateral circulation. C: Axial view showed portal vein and inferior vena cava thrombosis. Arrowheads indicate the lesions with EHPVO. Arrow indicates thrombotic lesions.

carcinomas can spread to the area adjacent to the inferior vena cava from common iliac lymph nodes or can involve the peritoneum/mesentery. Tully et al. (11) and our previous report (8) demonstrated a high incidence of vascular thromboembolic events in patients with urothelial carcinoma. Breast and lung cancer caused the EHPVOs related to distant metastatic lesions behind the pancreas in our cases.

EHPVO is an uncommon complication of major malignancies. These complications can be due to tumor external compression with or without venous thrombosis. All our patients had metastatic disease. EHPVO of urological malignancies related to their anatomical location with regional lymph nodes or peritoneal spread. The complication of pre-renal azotemia is not related to dehydration or drug toxicity. However. this abnormality is probably related to coagulopathy and cytokine production resulting in acute renal injury $(8,12)$. Gammaglutamyl transpeptidase can be used to help to detect liver disease but is non-specific (6). CT scans can help to detect lesions with obstructed levels $(13,14)$. Axial-view CT scans can show lesions behind the pancreas and anterior to the inferior vena cava and aorta. Coronal-view CT scans can detect lesions with obstructed levels. Thirty percent of our patients exhibited vascular thromboses (in the portal vein or inferior vena cava) on CT scans $(13,14)$. Our one case with a squamous cell carcinoma (Figure 4) exhibited collateral circulation formation.

The etiology of liver enzyme elevation is probably due to the tumor causing insufficient portal blood flow to the liver. Unlike portal vein hypertension, the collateral circulation is insufficient at the time of formation (15). Unlike ischemic hepatitis, the acute liver injury in these patients and reduced blood flow to the liver are not due to shock or low blood pressure (16).

Treatment principally aims to relieve the external compression and decreasing hypercoagulation and cytokine production. Two-thirds of the patients exhibited improved liver function after chemotherapy. LMWH therapy was administered in cases in which complications of coagulopathy occurred (17-20). Dexamethasone was used to suppress cytokine formation (18). However, EHPVO with hepatic enzyme elevation is usually a terminal disease, and the survival time was short.

Our study has several important limitations. Firstly, the data were collected from a retrospective case cohort study from a single center and mainly from one single physician. Secondly, the imaging did not include magnetic resonance angiography to examine correlations with vascular obstructions. Thirdly, the diagnoses lacked tissue-based confirmation

\section{Conclusion}

EHPVO occurred in patients with metastatic cancer, leading to elevation of hepatic enzyme levels, resembling hepatitis but without hepatitis risk factors and cirrhosis. EHPVO should be considered in differential diagnosis. Treating underlying disease by systemic therapy can improving liver function in selective cases. On the contrary, chemotherapy is needed to improve liver enzyme and tumor control.

\section{Funding}

The Authors received no financial support for the research, authorship and publication of this article.

\section{Authors' Contributions}

TL performed data analysis and article writing/editing; CL collected and the data collection and management; $\mathrm{HH}$ analyzed the data; $\mathrm{CH}$ offered cases and management; JWC offed important opinion; and YJ offered image knowledge; All Authors read and provided final approval of the article. 


\section{Ethics Approval and Consent to Participate}

The Authors declare that an Institutional Review Board/Ethics Committee determined that informed consent was not required.

\section{Consent for Publication}

Not applicable.

\section{Conflicts of Interest}

The Authors declare that they have no competing interests in regard to this study.

\section{Acknowledgements}

The Authors wish to thank the oncology nursing staff for providing the best possible care for the patients of this study.

\section{References}

1 Chung HM and Wong K: Gross Anatomy (Sixth Edition) Philadelphia: Wolters Kluwer Health/Lippincott Williams \& Wilkins, 2007.

2 Sarin SK and Agarwal SR: Extrahepatic portal vein obstruction. Semin Liver Dis 22: 43-58, 2002. PMID: 11928078.

3 Wani ZA, Bhat RA, Bhadoria AS and Maiwall R: Extrahepatic portal vein obstruction and portal vein thrombosis in special situations: Need for a new classification. Saudi J Gastroenterol 21: 129-138, 2015. PMID: 26021771. DOI: 10.4103/1319-3767. 157550

4 Kumar A, Sharma P and Arora A: Portal vein obstruction: Epidemiology, pathogenesis, natural history, prognosis and treatment. Aliment Pharmacol Ther 41: 276-2192, 2015. PMID: 25475582. DOI: 10.1111/apt.13019

5 Rangari M, Gupta R, Jain M, Malhotra V and Sarin SK: Hepatic dysfunction in patients with extrahepatic portal venous obstruction. Liver Int 23: 434-439. 2003. PMID: 14986818.

6 Johnston DE: Special considerations in interpreting liver function tests. Am Fam Physician 59: 2223-2230, 1999. PMID: 10221307.

7 Liao TY, Hsu HC, Wen MS, Juan YH, Hung YH and Liaw CC: Iliofemoral venous thrombosis mainly related to iliofemoral venous obstruction by external tumor compression in cancer patients. Case Rep Oncol 9: 760-771, 2016. PMID: 27990113. DOI: $10.1159 / 000452943$

8 Liaw CC, Chang H, Yang TS and Wen MS: Pulmonary venous obstruction in cancer patients. J Oncol 2015: 210916, 2015. PMID: 26425121. DOI: 10.1155/2015/210916

9 Schwarzbach CJ, Schaefer A, Ebert A, Held V, Bolognese M, Kablau M, Hennerici MG and Fatar M: Stroke and cancer: The importance of cancer-associated hypercoagulation as a possible stroke etiology. Stroke 43: 3029-3034, 2012. PMID: 22996958. DOI: $10.1161 /$ STROKEAHA.112.658625
10 Fearon KC, Voss AC and Husted DS: Cancer Cachexia Study Group. Definition of cancer cachexia: Effect of weight loss, reduced food intake, and systemic inflammation on functional status and prognosis. Am J Clin Nutr 83: 1345-1350, 2006. PMID: 16762946. DOI: 10.1093/ajcn/83.6.1345

11 Tully CM, Apolo AB, Zabor EC, Regazzi AM, Ostrovnaya I, Furberg HF, Rosenberg JE and Bajorin DF: The high incidence of vascular thromboembolic events in patients with metastatic or unresectable urothelial cancer treated with platinum chemotherapy. Cancer 122(5): 712-721, 2016. PMID: 26618338. DOI: $10.1002 /$ cncr.29801

12 Basile DP, Anderson MD and Sutton TA: Pathophysiology of acute kidney injury. Compr Physiol 2: 1303-1353, 2012. PMID: 23798302. DOI: 10.1002/cphy.c110041

13 Arora A and Sarin SK: Multimodality imaging of primary extrahepatic portal vein cancer obstruction (EHPVO): What every radiologist should know. Br J Radiol 88: 20150008, 2015. DOI: 10.1259/bjr.20150008

14 Pargewar SS, Desai SN, Rajesh S, Singh VP, Arora A and Mukund A: Imaging and radiological interventions in extrahepatic portal vein obstruction. World J Radiol 8: 556-570, 2016. PMID: 27358683. DOI: 10.4329/wjr.v8.i6.556

15 Berzigotti A, Seijo S, Reverter E and Bosch J: Assessing portal hypertension in liver diseases. Expert Rev Gastroenterol Hepatol 7: 141-155, 2013. PMID: 23363263. DOI: 10.1586/egh.12.83

16 Fuchs S, Bogomolski-Yahalom V, Paltiel O and Ackerman Z: Ischemic hepatitis: clinical and laboratory observations of 34 patients. Clin Gastroenterol 26: 183-186, 1998. PMID: 9600366.

17 Falang M, Marchetti A and Vignoli A: Coagulation and cancer: Biological and clinical aspects. J Thromb Haemost 11: 223-233, 2013. PMID: 23279708. DOI: 10.1111/jth.12075

18 El Azab SR, Rosseel PM, de Lange JJ, Groeneveld AB, van Strik R, van Wijk EM and Scheffer GJ: Dexamethasone decreases the pro- to anti-iinflammatory cytokine ratio during cardiac surgery. Br J Anaesth 88: 496-501, 2002. PMID: 12066724. DOI: $10.1093 / \mathrm{bja} / 88.4 .496$

19 Ettelaie C, Fountain D, Collier ME, Elkeeb AM, Xiao YP and Maraveyas A: Low molecular weight heparin downregulates tissue factor expression and activity by modulating growth factor receptor-mediated induction of nuclear factor- $\mathrm{kB}$. Biochim Biophys Acta 1812: 1591-1600, 2011. PMID: 21946214. DOI: 10.1016/j.bbadis.2011.09.007

20 Watson HG, Keeling DM, Laffan M, Tait RC and Makris M: British Committee for Standards in Haematology. Guideline on aspects of cancer-related venous thrombosis. Br J Haematol 170: 640-648, 2015. PMID: 26114207. DOI: 10.1111/bjh.13556 